\title{
Estado nutricional de mangueiras determinado pelos métodos DRIS e CND
}

\author{
Lucas S. Politi', Rilner A. Flores ${ }^{2}$, Juliana A. S. da Silva ${ }^{1}$, \\ Paulo G. S. Wadt ${ }^{3}$, Paulo A. da C. Pinto ${ }^{4}$ \& Renato de M. Prado ${ }^{1}$
}

\begin{abstract}
RESUMO
Diferentes procedimentos referentes do método do sistema integrado de diagnose e recomendação podem influenciar a acurácia da diagnose foliar da mangueira. Assim, o objetivo deste estudo foi comparar diagnósticos do estado nutricional da mangueira obtidos através de diferentes métodos de avaliação do estado nutricional, comparando-se relações multivariadas (método CND) com relações bivariadas (método DRIS), ou seja, com variações quanto ao uso de normas específicas ou preliminares e à transformação logarítmica dos dados. Foram utilizados resultados de análises de teores foliares de macro e micronutrientes de 63 pomares de mangueira do Vale do Submédio São Francisco. Para a interpretação dos índices nutricionais através do critério do Potencial de Resposta à Adubação. Os métodos CND e DRIS, com e sem transformação logarítmica e se usando normas específicas ou preliminares, as quais apresentaram desempenho semelhante na avaliação do estado nutricional da mangueira no Submédio São Francisco. Nos pomares de manga do semiárido nordestino as deficiências de micronutrientes ( $\mathrm{Zn}$, Fe e $\mathrm{Cu}$ ) foram mais frequentes que a deficiência de macronutrientes.
\end{abstract}

Palavras-chave: Mangifera indica, diagnose foliar, equilíbrio nutricional

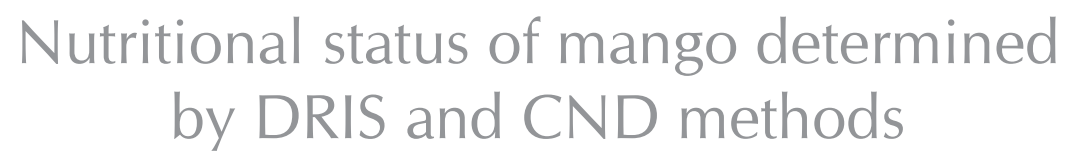

\begin{abstract}
Different procedures for the integrated method of diagnosis and recommendation may influence the accuracy of foliar diagnosis of mango. Thus, the objective of this study was to compare the diagnosis of nutritional status of mango obtained by different methods of assessing nutritional status, comparing multivariate relations (method CND) with bivariate relationships (DRIS) i.e., variations in the use of specific or preliminary standards and a logarithmic transformation of the data. The macro and micronutrient leaf analysis results of 63 mango orchards of Lower-middle São Francisco River Valley, Brazil, were used. To interpret nutritional status the Potential Response to Fertilization (PRA) criteria was used. The CND and DRIS methods, with and without logarithmic transformation and using specific or preliminary standards, which showed similar performance in assessing the nutritional status of the mango in Lower-middle San Francisco. Mango orchards in the semiarid region of northeast Brazil, the micronutrient deficiencies ( $\mathrm{Zn}$, Fe and $\mathrm{Cu}$ ) were more frequent than macronutrient deficiency.
\end{abstract}

Key words: Mangifera indica, foliar diagnosis, nutritional balance

\footnotetext{
1 Departamento de Solos e Adubo/Universidade Estadual Paulista, Via de Acesso Paulo Donato Castellane, s/n. CEP 14884-900, Jaboticabal, SP. Fone: (16) 3209-2672. Email: lucasspoliti@yahoo.com.br; jujuapsantos@hotmail.com; rmprado@fcav.unesp.br

${ }^{2}$ Escola de Agronomia e Engenharia de Alimentos/ UFG, Campus Samambaia, C.P. 131, CEP 74001-970, Goiânia, GO. E-mail: rilner1@hotmail.com

${ }^{3}$ Empresa Brasileira de Pesquisa Agropecuária - Embrapa Acre, Rod. BR 364, km 14, CEP 69900-056, Rio Branco, AC. Fone: (68) 3212-3218. E-mail: paulogswadt@dris.com.br

${ }^{4}$ Universidade do Estado da Bahia, Campus III, Av. Edgard Chastinet, s/n, CEP 48905-680, Juazeiro, BA. E-mail: pacostapinto@hotmail.com
} 


\section{INTRODUÇÃO}

$\mathrm{O}$ fruto da mangueira tem sido uma elevada demanda interna além de excelente e sempre crescente aceitação no mercado internacional, tornando a mangueira (Mangifera indica L.) uma das principais espécies frutíferas cultivadas no Brasil (Oliveira et al., 2002) em sistemas não irrigados na região sudeste (Blanco et al., 2004) e em sistemas irrigados na região nordeste do Brasil (Oliveira et al., 2002). As ferramentas mais utilizadas para o monitoramento nutricional de pomares de mangueiras consistem na análise de solos e na diagnose foliar. O uso da análise de solo como única ferramenta para recomendação de fertilizantes tem aplicabilidade limitada em espécies arbóreas frutíferas em razão da distribuição do sistema radicular, hábitos perenes, efeitos do porta-enxertos e da frutificação alternada (Bhargava \& Chadha, 1988).

O entendimento dos processos relacionados à nutrição das frutíferas é complexo e a identificação dos fatores limitantes da produtividade necessita isolar dos fatores nutricionais aqueles fatores não nutricionais, sendo comum que fatores ambientais e biológicos respondam por flutuações na produtividade das frutíferas mesmo quando não há nenhum fator nutricional envolvido (Hundal et al., 2005).

Em mangueiras (Mangifera indica $\mathrm{L}$.) a técnica da diagnose foliar adota dois métodos para a interpretação do estado nutricional das árvores: o nível crítico ou faixa de suficiência (NC) (Silva et al., 2002) e o Sistema Integrado de Diagnose e Recomendação (DRIS) (Pinto et al., 2009).

A diagnose foliar realizada pelo $\mathrm{NC}$ não considera interações com outros nutrientes e pode ser afetada por fatores não controlados, como a taxa de acúmulo de biomassa nos tecidos foliares (Wadt, 2008) tornando o método mais seguro em situações nas quais as condições de manejo dos pomares forem semelhantes às utilizadas na calibração do método.

Por sua vez pressupõe-se, com a utilização do DRIS, que os diagnósticos nutricionais tenham menor dependência a fatores ambientais não controlados (Wadt, 2008; Hundal et al., 2005) além de considerar a interação entre os nutrientes e, desta forma, tornar possível identificar a ordem de limitação dos nutrientes (Jones, 1981).

O método DRIS, todavia, suporta vários procedimentos distintos dentre os quais o uso de relações bivariadas, em que o equilíbrio relativo dos nutrientes pode ser inferido a partir de relações duais (N/P, $\mathrm{P} / \mathrm{K}, \mathrm{K} / \mathrm{Ca}, \mathrm{Ca} / \mathrm{Mg}$, por exemplo) na amostra a ser diagnosticada ou pelo uso de relações multivariadas, no método também conhecido como Diagnose da Composição Nutricional (CND) (Parent \& Dafir, 1992).

Alguns autores consideram que as relações bivariadas sejam uma melhor indicação do desequilíbrio nutricional (Bhupal \& Rao, 2006; Wadt \& Silva, 2010) enquanto outros preconizam o uso de relações multivariadas como a forma adequada para se avaliar o equilíbrio nutricional (Parent, 2011). Mesmo considerando as relações bivariadas diferentes procedimentos de cálculo produzem índices de diferentes valores, com possibilidades de se obter diferentes interpretações do estado nutricional de uma mesma lavoura (Silva et al., 2009) ou pomar como, por exemplo, o uso de diferentes normas DRIS (Partelli et al., 2006; Dias et al., 2010a) ou a adoção ou não de transformação logarítmica dos dados (Dias et al., 2010b).
Neste sentido, objetivou-se comparar diagnósticos do estado nutricional da mangueira obtidos pelo método CND e DRIS pela fórmula de Jones (1981) com variações devidas ao uso de diferentes normas e transformação logarítmica dos dados.

\section{Material e MÉTOdos}

Os dados utilizados foram obtidos de sessenta e três pomares comerciais de manga, variedade Tommy Atkins, da região do Vale do Submédio São Francisco, amostrados no período de 1997 a 1999 (Pinto et al., 2009). O clima da região é Bswh, com temperatura do mês mais frio superior a $18^{\circ} \mathrm{C}$, temperatura média anual de $26,3{ }^{\circ} \mathrm{C}$ e precipitação (no período de 1998 a 1999) média de 555,3 mm e evapotranspiração média de 1900 $\mathrm{mm}$ ano $^{-1}$, com umidade relativa variando de 45 a $80 \%$.

Obtiveram-se os dados de pomares com árvores de sete ou mais anos de idade, sem problemas sanitários aparentes e com porte uniforme. As amostras foram tomadas na semana anterior às pulverizações das copas com nitrato de potássio ou nitrato de cálcio, na porção mediana da copa e nos quatro pontos cardeais, de vinte e cinco árvores, totalizando cem folhas por pomar, as quais foram acondicionadas em sacos de papel e encaminhadas ao laboratório.

No laboratório as folhas foram lavadas em água destilada, colocadas em estufa com circulação mecânica de ar na temperatura variando de 65 a $70^{\circ} \mathrm{C}$ até peso constante, moídas em moinho Wiley e passadas em peneiras de $1 \mathrm{~mm}$ de malha. Nas amostras foliares foram determinados os teores totais de $\mathrm{N}, \mathrm{P}, \mathrm{K}, \mathrm{Ca}, \mathrm{Mg}, \mathrm{B}, \mathrm{Cu}, \mathrm{Fe}, \mathrm{Mn}$ e $\mathrm{Zn}$, conforme procedimentos descritos por Malavolta et al. (1997) e Pinto et al. (2009).

$\mathrm{Na}$ aplicação dos métodos diagnósticos foram utilizados dois grupos de normas: normas DRIS preliminares $(\mathrm{P})$ sugeridas pela Embrapa Semiárido (Wadt \& Silva, 2010) e obtidas de levantamento nutricional mensal em dez pomares de alta produtividade da região do Vale do Submédio São Francisco e normas DRIS específicas (E) obtidas das sessenta e três lavouras monitoradas neste trabalho.

Na obtenção dessas normas foram calculadas as médias e as variâncias de todas as relações bivariadas entre os nutrientes, para relações log-transformadas e sem a transformação logarítmica, resultando em quatro conjuntos de padrões nutricionais: Normas Preliminares Log-Transformadas (P L), Normas Preliminares Comuns (P C), Normas Específicas LogTransformadas (E L) e Normas Específicas Comuns (E C).

Para o grupo de normas específicas também se obtiveram normas para as relações multivariadas, conforme Parent \& Dafir (1992) denominadas Normas CND, equivalendo às normas específicas multivariadas.

Os índices DRIS foram calculados pelos seguintes procedimentos:

Para o procedimento com relações bivariadas os índices DRIS de cada nutriente foram calculados pela média aritmética de todas as funções DRIS, nas formas direta e inversa:

$\mathrm{I}(\mathrm{A})=[\mathrm{f}(\mathrm{A} / \mathrm{B})+\ldots+\mathrm{f}(\mathrm{A} / \mathrm{N})-\mathrm{f}(\mathrm{B} / \mathrm{A})-\ldots-\mathrm{f}(\mathrm{N} / \mathrm{A})] / 2 \mathrm{n}$, cujas funções $\mathrm{f}(\mathrm{A} / \mathrm{B})$ foram calculadas pelas seguintes fórmulas (Jones, 1981):

Fórmula de Jones: $\mathrm{f}(\mathrm{A} / \mathrm{B})=(\mathrm{A} / \mathrm{B}-\mathrm{a} / \mathrm{b}) / \sigma \mathrm{a} / \mathrm{b}$

Fórmula de Jones com relações log-transformadas: $\mathrm{f}(\mathrm{A} / \mathrm{B})$ $=(\log (\mathrm{A} / \mathrm{B})-\log (\mathrm{a} / \mathrm{b})) / \sigma \log (\mathrm{a} / \mathrm{b})$ 
Para o procedimento com relações multivariadas os índices CND foram obtidos pela expressão (Parent \& Dafir, 1992):

$$
I(A)=\frac{(Z-z)}{s}
$$

em que:

I(A) - índice DRIS ou índice CND de um nutriente A qualquer

$\mathrm{A}, \mathrm{B}$ e $\mathrm{N}$ - representam quaisquer nutrientes

$\mathrm{f}(\mathrm{A} / \mathrm{B})$ e $\mathrm{f}(\mathrm{A} / \mathrm{N})$ - funções DRIS na forma direta para o nutriente $A$

$\mathrm{f}(\mathrm{B} / \mathrm{A})$ e $\mathrm{f}(\mathrm{N} / \mathrm{A})$ - funções DRIS na forma indireta para o nutriente $\mathrm{A}$

$\mathrm{A} / \mathrm{B}$ e a/b, valor da relação e da média da mesma relação, respectivamente, em uma amostra e na população de referência tomada para a obtenção das normas DRIS

n - número de relações nutricionais utilizadas para o cálculo do índice DRIS

$\sigma_{\mathrm{a} / \mathrm{b}}$ - desvio padrão da relação $\mathrm{a} / \mathrm{b}$ na população de referência

$\mathrm{Z}$ e $\mathrm{z}$ - relação multivariada e respectiva média na população de referência de um nutriente qualquer

S - desvio padrão da relação multivariada na população de referência

As populações de referência foram representadas pelas estatísticas (média e variância) das normas preliminares e específicas, descritas acima.

O índice de balanço nutricional médio (IBNm) foi calculado pela média aritmética dos valores absolutos dos índices nutricionais (I(A)) gerados para cada amostra para todos os métodos diagnósticos utilizados. Com vista à interpretação dos índices DRIS utilizou-se o critério do Potencial de Resposta à Adubação (Wadt, 2005) com cinco categorias:

i) Equilíbrio nutricional $=|\mathrm{I}(\mathrm{A})|<\mathrm{IBNm}$. Atribuiu-se o valor zero ou a notação "z"

ii) Alta probabilidade de insuficiência $|\mathrm{I}(\mathrm{A})|>\mathrm{IBNm}, \mathrm{I}(\mathrm{A})$ $<0$ e I(A) sendo o de menor valor. Atribui-se o valor -2 ou a notação "p"

iii) Baixa probabilidade de insuficiência $|\mathrm{I}(\mathrm{A})|>\mathrm{IBNm}$, $\mathrm{I}(\mathrm{A})<0$ e I(A) não sendo o de menor valor. Atribui-se o valor -1 ou a notação "pz"

iv) Alta probabilidade de excesso $|\mathrm{I}(\mathrm{A})|>\mathrm{IBNm}$, Inut $>0 \mathrm{e}$ I(A) sendo o de maior valor. Atribui-se o valor 2 ou a notação "nz"

v) Baixa probabilidade de excesso $|\mathrm{I}(\mathrm{A})|>\mathrm{IBNm}, \mathrm{I}(\mathrm{A})>$ 0 e I(A) não sendo o de maior valor. Atribui-se o valor 1 ou a notação "nz"

Para maior síntese, as classes "p" e "pz" também foram agrupadas em limitantes por falta (LF), as classes " $n$ " e "nz" foram agrupadas em limitante pelo excesso (LE) e a classe de resposta nula $(\mathrm{z})$ foi renomeada como não limitante (NL) conforme Silva et al. (2004).

Com base na interpretação dos índices obtidos compararamse os resultados dos diagnósticos obtidos pelas fórmulas de Jones, com a combinação das normas DRIS preliminares ou específicas e com ou sem a transformação logarítmica e também com os diagnósticos produzidos pelo método CND.

Nessas comparações calculou-se o número de casos de diagnósticos em cada classe do estado nutricional ( $\mathrm{p}, \mathrm{pz}, \mathrm{z}, \mathrm{nz}$, $\mathrm{n}$; ou LE, LF, NL) discordantes, sendo este valor descontado do número de casos prováveis obtendo-se, assim, o grau de concordância (GC) para o estado nutricional baseado no Potencial de Resposta à Adubação $\left(\mathrm{GC}_{\text {pra }}\right)$ ou na simplificação proposta por Silva et al. (2004) $\left(\mathrm{GC}_{\mathrm{s}}\right)$ :

$$
\begin{aligned}
\mathrm{GC}_{\mathrm{pra}} & =100-[|\mathrm{p} 1-\mathrm{p} 2|+|\mathrm{pz} 1-\mathrm{pz} 2|+|\mathrm{z} 1-\mathrm{z} 2|+ \\
& +|\mathrm{nz} 1-\mathrm{nz} 2|+|\mathrm{n} 1-\mathrm{n} 2|] \times 100 / 126
\end{aligned}
$$

ou

$$
\begin{gathered}
\mathrm{GC}_{\mathrm{s}}=100-[|\mathrm{LF} 1-\mathrm{LF} 2|+|\mathrm{NL} 1-\mathrm{NL} 2|+|\mathrm{LE} 1-\mathrm{LE} 2|] \mathrm{x} \\
\mathrm{x} 100 / 126
\end{gathered}
$$

em que:

$|\mathrm{p} 1-\mathrm{p} 2|,|\mathrm{pz} 1-\mathrm{pz} 2|,|\mathrm{z} 1-\mathrm{z} 2|,|\mathrm{nz} 1-\mathrm{nz} 2|$ e $|\mathrm{n} 1-\mathrm{n} 2|$, valor absoluto da diferença do número de casos de diagnósticos em cada uma das classes do potencial de resposta à adubação e:

|LF1-LF2|, |NL1-NL2| e |LE1-LE2|, valor absoluto da diferença do número de casos de diagnósticos em cada uma das classes simplificadas do potencial de resposta a adubação

Foram testadas também as distribuições da frequência com que os nutrientes foram apontados em cada uma das classes do potencial de resposta à adubação, na forma normal (cinco classes de estado nutricional) ou simplificada (três classes de estado nutricional), pelo teste de Qui-Quadrado a nível de significância de $1 \%$, sendo testadas duas hipóteses nulas:

Teste 1 (T1), com a seguinte hipótese nula: as frequências observadas para o estado nutricional de cada nutriente em relação aos diferentes métodos diagnósticos não foram diferentes entre si.

Teste 2 (T2), com a seguinte hipótese nula: as frequências observadas para o estado nutricional de todos os nutrientes não foram diferentes entre si para um mesmo método de diagnóstico.

No primeiro teste (T1) buscou-se avaliar se os métodos diagnósticos afetaram a distribuição do estado nutricional para cada um dos nutrientes avaliados; sendo a hipótese nula verdadeira, isto significa que os diagnósticos testados foram independentes do método diagnóstico testado. Neste procedimento a frequência esperada foi calculada para cada nutriente, pela média aritmética do estado nutricional fornecida pelos cinco procedimentos de cálculos testados. No segundo teste (T2) buscou-se avaliar se a frequência com que os nutrientes foram classificados em cada uma das classes do estado nutricional para cada um dos métodos diagnósticos foi independente e, caso tenha sido verdadeira a hipótese nula, significa que os diagnósticos produzidos foram aleatórios.

\section{Resultados E DISCUSSÃO}

Diferentes métodos ou procedimentos para a obtenção de índices de estado nutricional mostraram-se coerentes quanto aos diagnósticos resultantes, com exceção do uso da fórmula 
de Jones (1981) com normas específicas log-transformadas (J E L) e unicamente para os nutrientes N e K (Tabela 1, teste de $\left.x^{2} \mathrm{~T} 1\right)$ tendência também observada para micronutrientes em que somente para Mn e com o uso da fórmula de Jones (1981) com normas preliminares log-transformadas ( $\mathrm{J}$ P L) foram detectadas diferenças na distribuição dos nutrientes em relação ao seu estado nutricional (Tabela 2, teste de $x^{2} \mathrm{~T} 1$ ).
Avaliando o grau de concordância (GC) para a interpretação do estado nutricional, verifica-se que em $98 \%$ dos casos o GC foi superior a 70 e em $89 \%$ dos casos o GC foi superior a 80 e em $51 \%$ dos casos o GC foi superior a 90\% (Tabela 3 ) indicando que, em termos gerais, todos os procedimentos de cálculo apresentaram diagnósticos relativamente semelhantes entre si.

Tabela 1. Frequência, em porcentagem, do potencial de resposta à adubação para os macronutrientes (exceto $S$ ) determinada pelos métodos DRIS e CND, em amostras de mangueiras coletadas em pomares do Submédio do São Francisco, nos anos agrícolas de 1997-1999

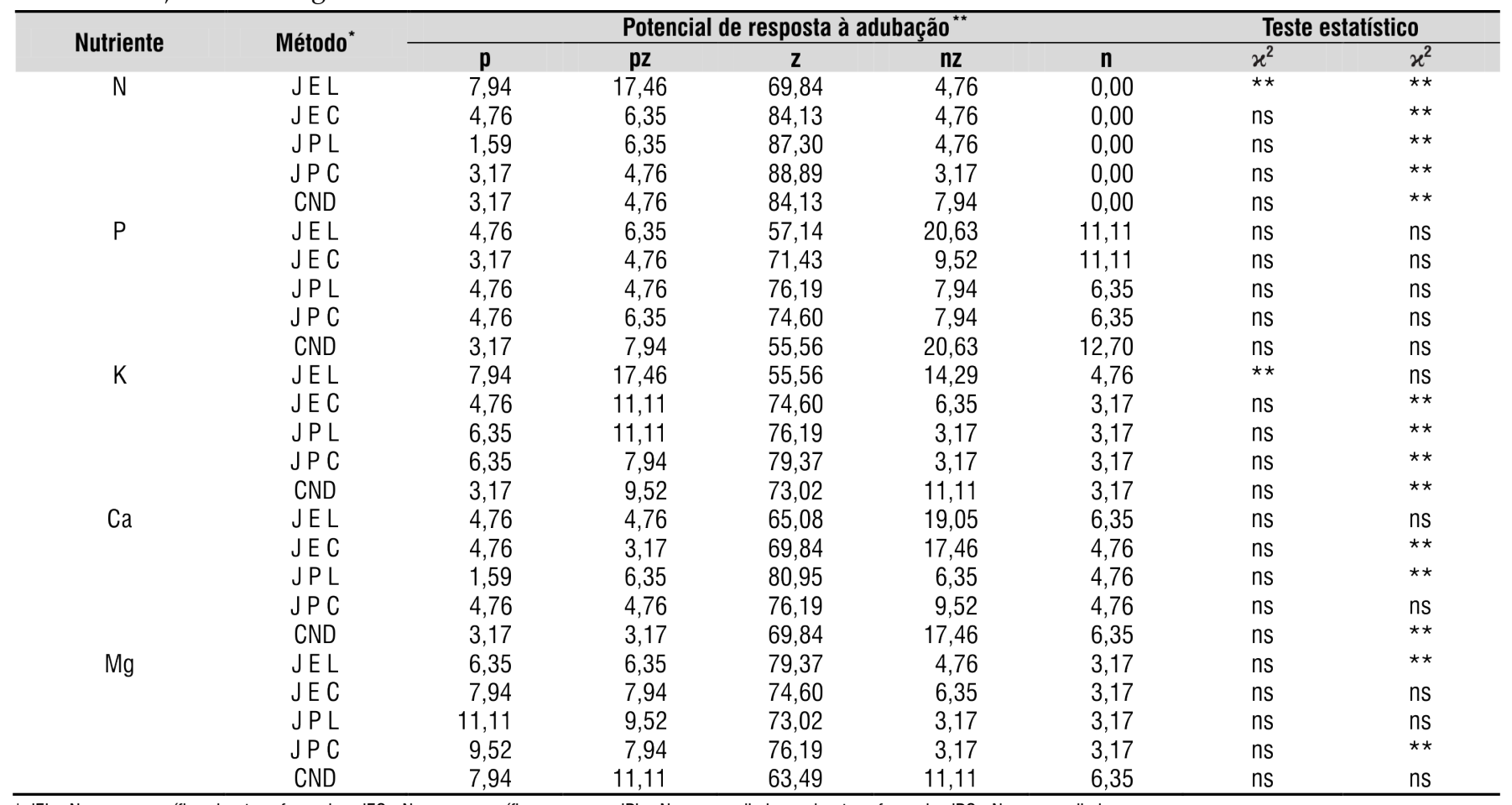

* JEL - Normas específicas log-transformadas; JEC - Normas específicas comuns; JPL - Normas preliminares log-transformada; JPC - Normas preliminares comuns

** p - positiva, com alta probabilidade; pz - positiva, com baixa probabilidade; $\mathrm{z}$ - nula; $\mathrm{nz}$ - negativa, com baixa probabilidade; $\mathrm{n}$ - negativa, com alta probabilidade

Tabela 2. Frequência, em porcentagem, do potencial de resposta à adubação para os micronutrientes, determinada pelos métodos DRIS e CND, em amostras de mangueiras coletadas em pomares do Submédio do São Francisco, nos anos agrícolas de 1997-1999

\begin{tabular}{|c|c|c|c|c|c|c|c|c|}
\hline \multirow{2}{*}{ Nutriente } & \multirow{2}{*}{ Método* } & \multicolumn{5}{|c|}{ Potencial de resposta à adubação ${ }^{\star \star}$} & \multicolumn{2}{|c|}{ Teste estatístico } \\
\hline & & $\mathbf{p}$ & $\mathrm{pz}$ & $Z$ & $\mathrm{nz}$ & $\mathbf{n}$ & $x^{2}$ & $x^{2}$ \\
\hline \multirow{3}{*}{ B } & JEC & 4,76 & 1,59 & 33,33 & 17,46 & 42,86 & ns & *夫 \\
\hline & JPC & 4,76 & 1,59 & 36,51 & 28,57 & 28,57 & ns & $\star \star$ \\
\hline & CND & 4,76 & 0,00 & 33,33 & 23,81 & 38,10 & ns & ** \\
\hline \multirow[t]{3}{*}{$\mathrm{Cu}$} & JEL & 22,22 & 17,46 & 44,44 & 6,35 & 9,52 & ns & $\star \star$ \\
\hline & JEC & 17,46 & 25,40 & 42,86 & 4,76 & 9,52 & ns & ** \\
\hline & CND & 19,05 & 19,05 & 47,62 & 3,17 & 11,11 & ns & $\star \star$ \\
\hline \multirow[t]{5}{*}{$\mathrm{Zn}$} & JEL & 12,70 & 30,16 & 52,38 & 4,76 & 0,00 & ns & ** \\
\hline & JEC & 23,81 & 26,98 & 42,86 & 4,76 & 1,59 & ns & ** \\
\hline & JPL & 25,40 & 20,63 & 47,62 & 6,35 & 0,00 & ns & ** \\
\hline & $J P C$ & 23,81 & 30,16 & 39,68 & 4,76 & 1,59 & ns & ** \\
\hline & CND & 26,98 & 19,05 & 47,62 & 4,76 & 1,59 & ns & $\star \star$ \\
\hline $\mathrm{Fe}$ & CND & 22,22 & 9,52 & 44,44 & 17,46 & 6,35 & ns & $\star \star$ \\
\hline
\end{tabular}

\footnotetext{
* JEL - Normas específicas log-transformadas; JEC - Normas específicas comuns; JPL - Normas preliminares log-transformada; JPC - Normas preliminares comuns
}

** p - positiva, com alta probabilidade; pz - positiva, com baixa probabilidade; $\mathrm{z}$ - nula; $\mathrm{nz}$ - negativa, com baixa probabilidade; $\mathrm{n}$ - negativa, com alta probabilidade 
Tabela 3. Frequência, em porcentagem, de talhões com diagnoses concordantes do Potencial de Resposta à Adubação (PRA) determinada pelos métodos DRIS e CND, em amostras foliares de manga coletadas em pomares do Submédio do São Francisco, nos anos agrícolas de 1997-1999

\begin{tabular}{|c|c|c|c|c|c|c|c|c|c|c|}
\hline \multirow{2}{*}{$\begin{array}{l}\text { Potencial de resposta } \\
\text { à adubação* }\end{array}$} & $\mathbf{N}$ & $\mathbf{P}$ & $\mathrm{K}$ & $\mathrm{Ca}$ & $\mathrm{Mg}$ & B & $\mathrm{Cu}$ & $\mathrm{Zn}$ & $\mathrm{Fe}$ & Mn \\
\hline & \multicolumn{10}{|c|}{$\%$} \\
\hline CND vs. J E C & 97 & 84 & 95 & 98 & 89 & 94 & 92 & 92 & 97 & 92 \\
\hline CND vs. J P C & 95 & 79 & 90 & 90 & 86 & 90 & 94 & 94 & 71 & 89 \\
\hline JEL vs. JEC & 86 & 86 & 81 & 95 & 95 & 98 & 92 & 98 & 98 & 87 \\
\hline JEL vs. JPL & 83 & 81 & 79 & 83 & 92 & 84 & 86 & 92 & 71 & 86 \\
\hline JEL vs. JPC & 81 & 83 & 76 & 89 & 95 & 84 & 90 & 92 & 73 & 87 \\
\hline JPL vs. JPC & 97 & 98 & 97 & 94 & 97 & 98 & 92 & 100 & 97 & 89 \\
\hline CND vs. J E C vs. JPC & 95 & 78 & 90 & 90 & 86 & 84 & 89 & 92 & 70 & 89 \\
\hline
\end{tabular}

* JEL - Normas específicas log-transformadas; JEC - Normas específicas comuns; JPL - Normas preliminares log-transformada; JPC - Normas preliminares comuns

Considerando-se os nutrientes individualmente, os diferentes procedimentos de cálculo apresentam elevado GC para o estado nutricional de $\mathrm{Zn}(100 \%$ dos casos com GC acima de 90\%), Ca, Mg, Cu e Mn (91\% dos casos com GC acima de $90 \%)$ e em menor intensidade para $\mathrm{N}$ e B $(73 \%$ dos casos com GC acima de 90\%); para os demais nutrientes, $\mathrm{K}, \mathrm{P}$, e principalmente $\mathrm{Fe}$, o GC foi menor (Tabela 3).

Comparando os métodos entre si verifica-se, independente do nutriente, que o uso da fórmula de Jones (1981) com as normas preliminares $(\mathrm{J} P)$ resultou em maior concordância em referência à utilização da transformação logarítmica (J P L) ou da não utilização deste recu rso de transformação logarítmica (J P C) com $90 \%$ dos nutrientes apresentando GC acima de $90 \%$ enquanto entre a fórmula de Jones (1981) usando-se normas específicas (J E) ou preliminares $(\mathrm{J} P$ ) foram observados apenas $20 \%$ de casos com GC acima de $90 \%$ (Tabela 3, J E L vs. J P L e J E L vs. J P C).

Trabalhos realizados em culturas anuais mostram resultados com menor grau de concordância. Por exemplo, Serra et al. (2010) constataram, avaliando a frequência de diagnoses concordantes em algodoeiro, que o $\mathrm{N}$ foi o nutriente que teve menor concordância entre os métodos DRIS e CND (70,8\%) sendo que, quanto aos demais nutrientes, a concordância esteve entre 73,8 e $87,7 \%$. Também em soja avaliando a frequência de diagnoses concordantes quanto ao PRA - Potencial de Resposta à Adubação, Urano et al. (2007) verificaram menor concordância entre os métodos avaliados para o $\mathrm{P}(74,6 \%)$ e para os outros nutrientes avaliados a frequência de diagnoses concordantes do PRA variou de 85,9 a 94,4\%.

Silva et al. (2005) também avaliaram, em eucalipto, a frequência de diagnoses concordantes do Potencial de Resposta à Adubação na região Centro-Leste do Estado de Minas Gerais sendo que, para os nutrientes N, P, K, Ca e Mg, essa concordância foi, em média, de $85 \%$ para o método DRIS e M-DRIS e de $84 \%$ para CND.

Quando se optou por classificar o estado nutricional em apenas três categorias (LF, LE e NL) tanto para os macronutrientes (Tabela 4) como para os micronutrientes (Tabela 5) o grau de concordância foi ainda maior (Tabela 6) situação diretamente decorrente da redução do número de classes de cinco para três, meramente reduzindo a possibilidade de classificações divergentes.
Tabela 4. Frequência de diagnose do estado nutricional para macronutrientes (exceto S), determinada pelos métodos DRIS e CND em amostras de mangueiras coletadas em pomares do Submédio do São Francisco, nos anos agrícolas de 1997-1999

\begin{tabular}{|c|c|c|c|c|c|c|}
\hline \multirow{2}{*}{ Nutriente } & \multirow{2}{*}{ Método* } & \multicolumn{3}{|c|}{ Estado nutricional $^{* *}$} & \multicolumn{2}{|c|}{ Teste estatístico } \\
\hline & & LF & NL & LE & $x^{2}$ & $x^{2}$ \\
\hline \multirow[t]{5}{*}{$\mathrm{N}$} & JEL & 25,40 & 69,84 & 4,76 & $\star \star$ & $\star \star$ \\
\hline & JEC & 11,11 & 84,13 & 4,76 & ns & ** \\
\hline & $J P L$ & 7,94 & 87,30 & 4,76 & ns & ** \\
\hline & JPC & 7,94 & 88,89 & 3,17 & ns & ** \\
\hline & CND & 7,94 & 84,13 & 7,94 & ns & $\star \star$ \\
\hline \multirow[t]{5}{*}{$P$} & JEL & 11,11 & 57,14 & 31,75 & ns & ns \\
\hline & JEC & 7,94 & 71,43 & 20,63 & ns & ** \\
\hline & $J P L$ & 9,52 & 76,19 & 14,29 & ns & $\star \star$ \\
\hline & $J P C$ & 11,11 & 74,60 & 14,29 & ns & ** \\
\hline & CND & 11,11 & 55,56 & 33,33 & ns & ns \\
\hline \multirow[t]{5}{*}{ K } & JEL & 25,40 & 55,56 & 19,05 & $\star \star$ & ns \\
\hline & JEC & 15,87 & 74,60 & 9,52 & ns & ** \\
\hline & $J P L$ & 17,46 & 76,19 & 6,35 & ns & ** \\
\hline & $J P C$ & 14,29 & 79,37 & 6,35 & ns & ** \\
\hline & CND & 12,70 & 73,02 & 14,29 & ns & ** \\
\hline \multirow[t]{5}{*}{$\mathrm{Ca}$} & JEL & 9,52 & 65,08 & 25,40 & ns & ** \\
\hline & JEC & 7,94 & 69,84 & 22,22 & ns & ** \\
\hline & $J P L$ & 7,94 & 80,95 & 11,11 & ns & ** \\
\hline & JPC & 9,52 & 76,19 & 14,29 & ns & $\star *$ \\
\hline & CND & 6,35 & 69,84 & 23,81 & ns & $\star \star$ \\
\hline \multirow[t]{5}{*}{$\mathrm{Mg}$} & JE L & 12,70 & 79,37 & 7,94 & ns & $\star *$ \\
\hline & JEC & 15,87 & 74,60 & 9,52 & ns & $\star *$ \\
\hline & JPL & 20,63 & 73,02 & 6,35 & ns & $* *$ \\
\hline & JPC & 17,46 & 76,19 & 6,35 & ns & $* *$ \\
\hline & CND & 19,05 & 63,49 & 17,46 & ns & ns \\
\hline
\end{tabular}

* JEL - Normas específicas log-transformadas; JEC - Normas específicas comuns; JPL - Normas preliminares log-transformada; JPC - Normas preliminares comuns

** LF - limitante por falta; NL - não limitante; LE - limitante por excesso

Para os macronutrientes e para a semelhança da interpretação obtida para o PRA, com exceção do uso da fórmula de Jones (1981) com normas específicas log-transformadas e unicamente para os nutrientes $\mathrm{N}$ e $\mathrm{K}$, não houve diferença no uso dos vários procedimentos quanto à distribuição dos nutrientes em função do estado nutricional determinado pelo DRIS (Tabela 4 , teste de $\varkappa^{2} \mathrm{~T} 1$ ); já para os micronutrientes todos os estados nutricionais determinados pelos diferentes procedimentos foram estatisticamente semelhantes entre si (Tabela 5, teste de $x^{2} \mathrm{~T} 1$ ).

Os nutrientes que apresentaram maior grau de concordância entre os métodos foram B e Zn (100\% de GC), $\mathrm{Ca}$ e $\mathrm{Mg}(91 \%$ de GC), Mg ( $82 \%$ de CG) e N e K (64\% de 
Tabela 5. Frequência, em porcentagem, de diagnose do estado nutricional para micronutrientes, determinada pelos métodos DRIS e CND em amostras de mangueiras coletadas em pomares do Submédio do São Francisco, nos anos agrícolas de 1997-1999

\begin{tabular}{|c|c|c|c|c|c|c|}
\hline \multirow{2}{*}{ Nutriente } & \multirow{2}{*}{ Método ${ }^{*}$} & \multicolumn{3}{|c|}{ Estado Nutricional $^{* *}$} & \multicolumn{2}{|c|}{ Teste estatístico } \\
\hline & & LF & NL & LE & $x^{2}$ & $x^{2}$ \\
\hline \multirow[t]{5}{*}{$B$} & JE L & 6,35 & 31,75 & 61,90 & ns & $\star \star$ \\
\hline & $\mathrm{JEC}$ & 6,35 & 33,33 & 60,32 & ns & ** \\
\hline & $J P L$ & 4,76 & 36,51 & 58,73 & ns & $\star *$ \\
\hline & $\mathrm{JPC}$ & 6,35 & 36,51 & 57,14 & ns & $\star \star$ \\
\hline & CND & 4,76 & 33,33 & 61,90 & ns & ** \\
\hline \multirow[t]{5}{*}{$\mathrm{Cu}$} & JEL & 39,68 & 44,44 & 15,87 & ns & ** \\
\hline & JEC & 42,86 & 42,86 & 14,29 & ns & ** \\
\hline & $J P L$ & 26,98 & 58,73 & 14,29 & ns & ns \\
\hline & $\mathrm{JPC}$ & 33,33 & 52,38 & 14,29 & ns & $\star \star$ \\
\hline & CND & 38,10 & 47,62 & 14,29 & ns & $\star \star$ \\
\hline \multirow[t]{5}{*}{$\mathrm{Zn}$} & JEL & 42,86 & 52,38 & 4,76 & ns & $\star \star$ \\
\hline & $\mathrm{JEC}$ & 50,79 & 42,86 & 6,35 & ns & $\star \star$ \\
\hline & $J P L$ & 46,03 & 47,62 & 6,35 & ns & ** \\
\hline & JPC & 53,97 & 39,68 & 6,35 & ns & $\star \star$ \\
\hline & CND & 46,03 & 47,62 & 6,35 & ns & $\star \star$ \\
\hline \multirow[t]{5}{*}{$\mathrm{Fe}$} & JEL & 36,51 & 42,86 & 20,63 & ns & $\star \star$ \\
\hline & $\mathrm{JEC}$ & 36,51 & 42,86 & 20,63 & ns & $\star *$ \\
\hline & $\mathrm{JPL}$ & 30,16 & 47,62 & 22,22 & ns & $* *$ \\
\hline & JPC & 30,16 & 47,62 & 22,22 & ns & ns \\
\hline & CND & 31,75 & 44,44 & 23,81 & ns & $\star \star$ \\
\hline \multirow[t]{5}{*}{$\mathrm{Mn}$} & JEL & 12,70 & 39,68 & 47,62 & ns & $\star \star$ \\
\hline & JEC & 11,11 & 44,44 & 44,44 & ns & $\star \star$ \\
\hline & JPL & 20,63 & 26,98 & 52,38 & ns & $\star *$ \\
\hline & $\mathrm{JPC}$ & 20,63 & 25,40 & 53,97 & ns & $\star *$ \\
\hline & CND & 11,11 & 44,44 & 44,44 & ns & ** \\
\hline
\end{tabular}

* JEL - Normas específicas log-transformadas; JEC - Normas específicas comuns; JPL - Normas preliminares log-transformada; JPC - Normas preliminares comuns

${ }_{\star *} \mathrm{LF}$ - limitante por falta; NL - não limitante; LE - limitante por excesso

GC) e com menor GC foram o P ( $55 \%$ de GC) e o Fe $(36 \%$ de GC) (Tabela 6).

Em geral, este comportamento foi distinto daquele observado pelo método do PRA como, por exemplo, para Ca, B e $\mathrm{Mn}$, que apresentaram um $\mathrm{GC}$ de $45 \%$, ou $\mathrm{Mg}$, que apresentou um GC de 55\%; entretanto, ao adotar a simplificação nas classes do estado nutricional perde-se um dos principais benefícios do sistema DRIS, isto é, o de identificar, pela ordem de limitação, os nutrientes mais limitantes por deficiência e por excesso.

Na comparação dos métodos entre si a maior semelhança entre os diagnósticos foi observada com o uso da fórmula de Jones (1981) e normas preliminares, com e sem transformação logarítmica (Tabela 6) e o menor GC foi constatado com o uso da fórmula de Jones (1981), com transformação logarítmica entre as normas preliminares e específicas (J P L e J E L) (Tabela 6). Partelli et al. (2006) observaram, ao comparar o diagnóstico produzido por normas DRIS de cafeeiros convencional e orgânicos, que esses diferiram segundo o tipo de norma utilizada.

As diferenças entre as normas DRIS podem ser minimizadas à medida que se aumenta a representatividade de cada norma em relação à população que se deseja avaliar como, por exemplo, em cupuaçueiros, quando Dias et al. (2010a) relataram semelhança no diagnóstico dos índices DRIS quando compararam normas DRIS específica com norma DRIS mais genérica, sendo esta composta de uma população de plantas que representa adequadamente a condição específica que se pretende avaliar.

Do ponto de vista da capacidade do sistema em identificar nutrientes limitantes à produtividade, torna-se imprescindível verificar se os diferentes procedimentos de cálculo dos índices DRIS e os critérios de interpretação resultam em distribuição não aleatória para a distribuição dos pomares em referência ao estado nutricional.

Neste sentido, apenas para $\mathrm{P}$ as distribuições observadas em todos os procedimentos testados, e para $\mathrm{Mg}$, na maioria dos procedimentos, foi aleatória (Tabela 1 , teste de $\varkappa^{2}$ T2) o que poderia estar sinalizando incapacidade do sistema em identificar o estado nutricional correto dos pomares para os referidos nutrientes. Este problema não foi observado para os demais macronutrientes e também não ocorreu quando houve redução do número de classes para a definição do estado nutricional dos pomares (Tabelas 2, 4 e 5, teste de $x^{2} \mathrm{~T} 2$ ).

Uma vez considerado que, em geral, os métodos se apresentaram com distribuições de frequência para o estado nutricional dos pomares estatisticamente semelhantes pelo teste de $\varkappa^{2}$ a $1 \%$ de significância (Tabelas $1,2,4$ e 5 , teste de $x^{2}$ T1) e se considerando que a distribuição de frequência para o estado nutricional dos nutrientes foi, em sua grande maioria, não casual (Tabelas 1, 2, 4 e 5, teste de $\varkappa^{2} \mathrm{~T} 2$ ) pode-se utilizar a média geral do estado nutricional proporcionado pelos diferentes métodos para se inferir sobre o estado nutricional dos pomares de manga monitorados (Tabela 7) optando-se por utilizar, neste momento, as cinco classes para identificação do Potencial de Resposta à Adubação em detrimento da simplificação em três classes já que a segunda alternativa é apenas uma síntese do mesmo critério de interpretação e deixa de enfatizar a importância

Tabela 6. Frequência de talhões, em porcentagem, com diagnoses concordantes do estado nutricional (EN), determinadas pelos métodos DRIS e CND, em amostras foliares de manga coletadas em pomares do Submédio do São Francisco, nos anos agrícolas de 1997-1999

\begin{tabular}{|c|c|c|c|c|c|c|c|c|c|c|}
\hline Estado nutricional ${ }^{\star}$ & $\mathbf{N}$ & $\mathbf{P}$ & K & Ca & Mg & B & Cu & $\mathrm{Zn}$ & $\mathrm{Fe}$ & $\mathbf{M n}$ \\
\hline CND vs. J E L & 84 & 99 & 85 & 96 & 89 & 98 & 98 & 97 & 97 & 96 \\
\hline CND vs. JE C & 98 & 90 & 98 & 99 & 93 & 99 & 95 & 97 & 100 & 95 \\
\hline CND vs. JPL & 98 & 89 & 96 & 94 & 94 & 98 & 89 & 98 & 87 & 100 \\
\hline CND vs. J P C & 98 & 90 & 96 & 95 & 93 & 98 & 95 & 98 & 86 & 92 \\
\hline JEL Vs. JEC & 86 & 91 & 86 & 97 & 96 & 99 & 98 & 100 & 97 & 91 \\
\hline J E L vs. JPL) & 83 & 90 & 86 & 91 & 93 & 97 & 87 & 94 & 90 & 96 \\
\hline JEL vs. JPC & 82 & 91 & 83 & 94 & 96 & 98 & 93 & 94 & 89 & 88 \\
\hline J E C vs. JPL & 97 & 97 & 98 & 94 & 97 & 98 & 84 & 94 & 87 & 95 \\
\hline JEC Vs. JPC & 96 & 97 & 97 & 96 & 98 & 98 & 90 & 94 & 86 & 97 \\
\hline JPL vs. JPC & 99 & 98 & 97 & 97 & 97 & 99 & 94 & 100 & 99 & 92 \\
\hline CND vs. J E C vs. J P C & 95 & 79 & 92 & 90 & 86 & 96 & 90 & 94 & 87 & 92 \\
\hline
\end{tabular}

* JEL - Normas específicas log-transformadas; JEC - Normas específicas comuns; JPL - Normas preliminares log-transformada; JPC - Normas preliminares comuns 
da ordem de limitação, uma informação relevante que, na cultura da mangueira, tem-se mostrado bastante significativa por permitir a correção das deficiências com maior segurança (Hundal et al., 2005).

Tabela 7. Frequência média, em porcentagem, para o Potencial de Resposta à Adubação em pomares de mangueiras do Submédio do São Francisco, nos anos agrícolas de 1997-1999

\begin{tabular}{crrrrr}
\hline \multirow{2}{*}{ Nutriente } & \multicolumn{5}{c}{ Potencial de resposta à adubação } \\
\cline { 2 - 6 } N & $\mathbf{p}$ & $\mathbf{p z}$ & $\mathbf{z}$ & $\mathbf{n z}$ & $\mathbf{n}$ \\
$\mathrm{P}$ & 4,1 & 7,9 & 82,9 & 5,1 & 0,0 \\
$\mathrm{~K}$ & 4,1 & 6,0 & 67,0 & 13,3 & 9,5 \\
$\mathrm{Ca}$ & 5,7 & 11,4 & 71,7 & 7,6 & 3,5 \\
$\mathrm{Mg}$ & 3,8 & 4,4 & 72,4 & 14,0 & 5,4 \\
$\mathrm{~B}$ & 8,6 & 8,6 & 73,3 & 5,7 & 3,8 \\
$\mathrm{Cu}$ & 4,8 & 1,0 & 34,3 & 23,2 & 36,8 \\
$\mathrm{Zn}$ & 17,5 & 18,7 & 49,2 & 5,1 & 9,5 \\
$\mathrm{Fe}$ & 22,5 & 25,4 & 46,0 & 5,1 & 1,0 \\
$\mathrm{Mn}$ & 21,0 & 12,1 & 45,1 & 14,0 & 7,9 \\
\hline & 7,6 & 7,6 & 36,2 & 26,0 & 22,5 \\
\hline
\end{tabular}

p - positiva, com alta probabilidade; pz - positiva, com baixa probabilidade; $\mathrm{z}$ - nula; $\mathrm{nz}$ - negativa, com baixa probabilidade; $n$ - negativa, com alta probabilidade

Adotando-se o critério acima descrito, $\mathrm{Zn}$, Fe e $\mathrm{Cu}$ foram os nutrientes com maior probabilidade de resposta à adubação, respectivamente, sendo os nutrientes mais limitantes em 22,5, 21,0 e $17,5 \%$ das lavouras. Quando considerada a reunião das classes de alta e média probabilidade de resposta à adubação (p e pz), teve-se $47,9 \%$ dos pomares monitorados com probabilidade de estarem deficientes em $\mathrm{Zn}$.

Os micronutrientes Fe e Cu não são considerados relevantes em sistemas de recomendação de adubação no semiárido irrigado embora possam ser utilizados em fertirrigações na forma de sulfatos ou quelatos (Silva et al., 2002). Entretanto, na avaliação do estado nutricional de pomares de Tommy Aktins pelo método DRIS, Schaffer et al. (1988) relatam que, isoladamente ou em conjunto, $\mathrm{Mn}$ e $\mathrm{Fe}$, foram os nutrientes mais relacionados à perda de produtividade dos pomares da Flórida, EUA.

Salienta-se que a variabilidade do teor de Fe nas árvores de manga exige que em cada pomar devem ser amostradas no mínimo 30 plantas, nos quatro pontos cardeais de cada árvore (120 folhas por pomar) para se minimizar a variabilidade amostral (Rozane et al., 2007) o que também poderia contribuir, em parte, para explicar prováveis erros na definição do estado nutricional para $\mathrm{Fe}$ em função de maior erro amostral para este nutriente.

A deficiência de $\mathrm{Zn}$ também parece refletir no número de pomares com excesso de $\mathrm{P}$, que chega a atingir $22,8 \%$ dos pomares, o que sugere um possível desbalanço na nutrição de $\mathrm{P}$ e $\mathrm{Zn}$, nutrientes com antagonismo conhecido em várias culturas (Marschner, 1995). Bhupal \& Rao (2006) relataram que em experimentos de adubação de longa duração $\mathrm{Fe}$ e $\mathrm{Zn}$ foram indicados como os nutrientes mais limitantes pelo método DRIS nos tratamentos de maior produtividade enquanto o $\mathrm{K}$ esteve como o mais limitante no tratamento de menor produtividade.

Wadt \& Silva (2010) verificaram que a fórmula de Jones (1981) apresentou elevada utilidade para a avaliação do estado nutricional de pomares de mangueiras resultando em baixo número de falsos diagnósticos para insuficiência e deficiência quando adotado o método do nível crítico como referência para o estado nutricional. Considerando-se a elevada utilidade desta fórmula infere-se, portanto, que nesses pomares a deficiência de micronutrientes seja mais limitante para a produtividade que a disponibilidade de macronutrientes.

No tocante ao excesso de nutrientes destaca-se o elevado número de pomares com excesso de boro $(36,8 \%$ com alta probabilidade de resposta negativa e $23,2 \%$ com média probabilidade de resposta negativa) e de Mn (26,0 e 22,5\%, para alta e média probabilidade de resposta negativa, respectivamente). Especialmente para B, Wadt \& Silva (2010) indicam que tanto a fórmula de Jones (1981) como o método CND (Parent \& Dafir, 1992) apresentam baixo número de falsos diagnósticos para situações de excesso e toxicidade em pomares de mangueiras, o que vem reforçar a possibilidade de haver desequilíbrios na nutrição deste micronutriente. Na literatura se ressalta para o cuidado que se deve tomar nas adubações com boro dada à proximidade entre o limite de deficiência e de toxicidade (Silva et al., 2002).

No mesmo sentido Hundal et al. (2005) relatam que o DRIS indicou deficiência em $\mathrm{P}$ entre os macronutrientes e em Mn e B, entre os micronutrientes, em pomares não fertilizados formados sobre solos aluviais no nordeste da Índia. Tais resultados são coerentes com as taxas de exportação de micronutrientes observadas nos frutos de mangueira do sistema de fertirrigação no semiárido nordestino: $\mathrm{Cu}$ e $\mathrm{Zn}$ são os nutrientes mais exportados pelos frutos das mangueiras, após o Fe enquanto o B é o menos exportado (Silva et al., 2002).

Com respeito ao baixo número de pomares $\mathrm{N}$ deficientes ( $12 \%$ com alta e média probabilidade de resposta positiva) (Tabela 7) este resultado foi coerente com ensaios de adubação conduzidos por Costa et al. (2011) que encontraram resposta quadrática a $\mathrm{N}$ em mangueiras da variedade Tommy Aktins, no município de Ipanguaçu, RN, sendo a resposta máxima obtida com baixas aplicações de nitrogênio. No mesmo trabalho não foi encontrada resposta da mangueira à adubação potássica, o que também está de acordo com o baixo número de pomares considerados responsivos com alta e média probabilidade de resposta.

É importante, ainda, destacar que, normalmente, se observa maior incidência de colapso interno em frutos de pomares de mangueira que receberam elevadas doses de nitrogênio (McKenzie, 1994). Baixos teores foliares de cálcio também estão associados com a incidência de colapso interno (Assis et al., 2004; Cracknell et al., 2004).

\section{Conclusões}

1. Os métodos CND e DRIS com e sem transformação logarítmica e se usando normas específicas ou preliminares, apresentaram desempenho semelhante na avaliação do estado nutricional da mangueira no Submédio São Francisco.

2. Nos pomares de manga do semiárido nordestino as deficiências de micronutrientes $(\mathrm{Zn}, \mathrm{Fe}$ e $\mathrm{Cu}$ ) foram mais frequentes que a deficiência de macronutrientes. 


\section{LITERATURA CITADA}

Assis, J. S.; Silva, D. J.; Moraes, P. L. D. Equilíbrio nutricional e distúrbios fisiológicos em manga 'Tommy Atkins'. Revista Brasileira de Fruticultura, v.26, p.326-329, 2004.

Bhargava, B. S.; Chadha, K. L. Leaf nutrient guide for fruit and plantation crops. Fertilizer Industry News, v.33, p.2129, 1988.

Bhupal, G. R.; Rao, A. P. Identification of yield-limiting nutrients in mango through DRIS indices. Communications in Soil Science and Plant Analysis, v.37, p.1761-1774. 2006.

Blanco, F. F.; Machado, C. C.; Coelho, R. D.; Folegatti, M. V. Viabilidade econômica da irrigação da manga para o Estado de São Paulo. Revista Brasileira de Engenharia Agrícola e Ambiental, v.8, p.153-159, 2004.

Costa, M. E.; Caldas, A. V. C.; Oliveira, A. F. G.; Gurgel, M. T.; Silva, R. M. Caracterização nutricional da mangueira "Tommy Atkins" em função da adubação nitrogenada. Agropecuária Científica no Semi-Árido, v.7, p.16-22, 2011.

Costa Pinto, P. A. da; Dias, L. E. ; Alvarez V., V. H.; Choudhury, M. M.; Vieira, G. . Avaliação de estado nutricional da mangueira Tommy Atkins no Submédio do Vale do Rio São Francisco: Estabelecimento das normas DRIS. Recursos Rurais, v.5, p.5-13, 2009.

Cracknell, T. A.; Cid, M. C.; Socorro, A. R.; Fernandez, D.; Rossel, P.; Galan, V. Effects of nitrogen and calcium supply on the incidence of internal fruit breakdown in 'Tommy Atkins' mangoes (Mangifera indica L.) grown in a soilless system. Acta Horticulturae, v.645, p.387-393, 2004.

Dias, J. R. M.; Perez, D. V.; Silva, L. M.; Lemos, C. O.; Wadt, P. G. S. Normas DRIS para cupuaçuzeiro cultivado em monocultivo e em sistemas agroflorestais. Pesquisa Agropecuária Brasileira, v.45, p.64-71, 2010a.

Dias, J. R. M.; Wadt, P. G. S.; Lemos, C. O.; Delarmelinda, E. A.; Solino, J. S.; Tavella, L. B. Relações nutricionais logtransformadas para avaliação nutricional de cupuaçueiros comerciais. Acta Amazônia. v.40, p.37-42, 2010b.

Hundal, H.S.; Singh, D.; Brar, J.S. Diagnosis and recommendation integrated system for monitoring nutrient status of mango trees in submountainous area of Punjab, India. Communiations in Soil Science and Plant Analysis, v.36, p.2085-2099, 2005.

Jones, C. A. Proposed modifications of the diagnosis and recommendation integrated system (DRIS) for interpreting plant analysis. Communications in Soil Science and Plant Analysis, v.12, p.785-974, 1981.

Malavolta, E.; Vitti, G. C.; Oliveira, S. A. Avaliação do estado nutricional das plantas-princípios e aplicações. 2.ed. Piracibaca; Potafos, 1997. 319p.

Marschner, H. Mineral nutrition of higher plant. 2.ed. New York: Academy, 1995. 889p.

McKenzie, C.B. The background skin colour of exported mango fruit in relation to tree nitrogen status. South African Mango Growers' Association Yearbook, v.14, p.26-28, 1994.

Oliveira, F. das C.; Coelho, E. F.; Vasconcelos, L. F. L.; Araújo, E. C. E. Produção de manga sob diferentes regimes de irrigação, em condições subúmidas. Revista Brasileira de Engenharia Agrícola e Ambiental, v.6, p.390-396, 2002.
Parent, L.E. Diagnosis of the nutrient compositional space of fruit crops. Revista Brasileira de Fruticultura, v.33, p.321334, 2011.

Parent, L. E.; Dafir, M. A theoretical concept of compositional nutrient diagnosis. Journal of the American Society for Horticultural Science, v.117, p.219-242, 1992.

Partelli, F. B.; Vieira, H. D.; Monnerat, P. H.; Viana, A. P. Comparação de dois métodos DRIS para o diagnóstico de deficiências nutricionais do cafeeiro. Pesquisa Agropecuária Brasileira, v.41, p.301-306, 2006.

Rozane, D. E.; Natale, W.; Prado, R. M.; Barbosa, J. C. Amostragem para diagnose do estado nutricional de mangueiras. Revista Brasileira de Fruticultura, v.29, p.371376, 2007.

Schaffer, K.; Larson, K.; Snyder, G. H.; Sanchez, C. A. Rectification of mineral deficiency associated with mango decline by DRIS. Horticultural Science, v.23, p.617-619. 1988.

Serra, A. P.; Marchetti, M. E.; Vitorino, A. C. T.; Novelino, J. O.; Camacho, M. A. Desenvolvimento de normas Dris e CND e avaliação do estado nutricional da cultura do algodoeiro. Revista Brasileira de Ciência do Solo, v.34, p.97-104, 2010.

Silva, D. J.; Quaggio, J. A.; Pinto, P. A. de C.; Pinto, A. C. de Q.; Magalhães, A. F. de J. Nutrição e adubação. In: Genú, P. J. C.; Pinto, A. C. de Q. (Ed.). A cultura da mangueira. Brasília: Embrapa Informação Tecnológica, 2002. p.191-221.

Silva, G. G. C.; Neves, J. C. L.; Alvarez, V. H. V.; Leite, F. P. Nutritional diagnosis for eucalypt by DRIS, M-DRIS, and CND. Scientia Agricola, v. 61, p.507-515, 2004.

Silva, G. G. C.; Neves, J. C. L.; Alvarez V., V. H.; Leite, F. P. Avaliação da universalidade das normas DRIS, M-DRIS e CND. Revista Brasileira de Ciência do Solo, v.29, p.755761, 2005.

Silva, M. A. C.; Natale, W.; Malheiros, E. B.; Pavinato, A. Estabelecimento e avaliação de normas DRIS para a cultura do algodão no centro-oeste do Brasil. Acta Scientiarum. Agronomy, v.31, p.93-99, 2009.

Urano, E. O. M.; Kurihara, C. H.; Maeda, S.; Vitorino, A. C. T.; Gonçalves, M. C.; Marchetti, M. E. Determinação de teores ótimos de nutrientes em soja pelos métodos chance matemática, sistema integrado de diagnose e recomendação e diagnose da composição nutricional. Revista Brasileira de Ciência do Solo, v.31, p.63-72, 2007.

Wadt, P. G. S. Relationships between soil class and nutritional status of coffee plantations. Revista Brasileira de Ciência do Solo, v.29, p.277-234. 2005.

Wadt, P. G. S. Análise foliar para recomendação de adubação em culturas agrícolas. In: Prado; R. M.; Rozane, D. E.;Vale, D. W. do; Correia, M. A. R.; Souza, H. A. de. (Org.). Nutrição de plantas. Diagnose foliar em grandes culturas. Jaboticabal: Fundenesp, 2008, v.1, p.115-133.

Wadt, P. G. S.; Silva, D. J. Acurácia do diagnóstico nutricional de pomares de mangueiras obtido por três fórmulas DRIS. Revista Brasileira de Pesquisa Agropecuária, v.45, p.11801188. 2010. 